

\section{EVALUACIÓN DEL BLOQUE DE TIERRA MEDIANTE UN ESTUDIO DE VIABILIDAD CONSTRUCTIVA (EVC)}

\section{ASSESSMENT OF EARTH BLOCKS BY MEANS OF A CONSTRUCTION FEASIBILITY STUDY (CFS)}

\author{
ANA ROMERO GIRÓN \\ Doctora en Arquitectura \\ Investigadora, Departamento de Construcciones Arquitectónicas I \\ Universidad de Sevilla, Escuela Técnica Superior de Arquitectura \\ Sevilla, España \\ https://orcid.org/0000-0002-0536-4458 \\ anaromerogiron@gmail.com \\ MARIA REYES RODRÍGUEZ GARCÍA \\ Doctora en Química \\ Profesor titular, Departamento de Construcciones Arquitectónicas I \\ Universidad de Sevilla, Escuela Técnica Superior de Arquitectura \\ Sevilla, España \\ https://orcid.org/0000-0002-8331-2303 \\ rgarcia@us.es
}

\author{
JACINTO CANIVELL \\ Doctor en Arquitectura \\ Profesor Contratado Departamento de Construcciones Arquitectónicas II \\ Universidad de Sevilla, Escuela Técnica Superior de Ingeniería de \\ Edificación, Sevilla, España \\ https://orcid.org/0000-0001-7636-102X \\ jacanivell@us.es \\ ANA GONZALEZ SERRANO \\ DOCTORA EN ARQUITECTURA \\ Profesora Colaboradora, Departamento de Construcciones \\ Arquitectónicas IUniversidad de Sevilla, Escuela Técnica Superior \\ de ArquitecturaSevilla, España \\ https://orcid.org/0000-0001-5104-8555 \\ gserrano@us.es
}

\section{RESUMEN}

Aunque la construcción con bloques de tierra (BT) está avalada por numerosos trabajos científicos, existe una desconfianza sobre su viabilidad constructiva, agravada por la falta de formación técnica específica. Ante esta incertidumbre, muy presente en el ámbito español, es preciso dar respuestas técnicas fundamentadas. En esa dirección, este artículo expone el diseño y validación de una herramienta para la evaluación de la viabilidad constructiva del BT. Con ese fin, se seleccionan 29 casos de estudio en España, con los que se establecen las determinaciones constructivas y los indicadores para la evaluación de un grado de idoneidad técnica. Este parámetro, como resultado de la herramienta propuesta, sirve como apoyo a la toma de decisiones, la mejora del diseño y la eficiencia de las soluciones que emplean BT. Se concluye con la validación de la herramienta que demuestra su fiabilidad y adaptabilidad a cualquier situación. Finalmente, a partir del análisis de casos, se expone cómo la calidad del producto unida a condiciones externas adversas, aún con diseños constructivos correctos, define una situación común por la que el grado de idoneidad de la solución es reducida. Por lo tanto, es necesario exigir también productos con avales y prescripciones que garanticen y ofrezcan suficiente seguridad técnica.

Palabras clave

construcción sostenible, materiales tradicionales, bioconstrucción, cerramiento de la edificación

\section{ABSTRACT}

Although earth block construction (EB) is supported by numerous scientific works, there is a lack of confidence in its constructive viability, aggravated by the lack of specific technical training. In view of this uncertainty, which is widespread in Spain, it is necessary to provide well-founded technical responses.

This article, considering these aspects, presents the design and validation of a tool to assess the constructive viability of EB. For this purpose, 29 case studies are chosen in Spain, which establish the constructive use determinations and indicators to assess a degree of technical suitability. This parameter, as a result of the proposed tool, acts as a support for decision-making, the improvement of the design and, the efficiency of the solutions that use EB. It concludes by validating the tool, demonstrating its reliability and adaptability to any situation. Finally, the case analysis shows how the quality of the product combined with adverse external conditions, even with correct construction designs, defines a common

situation where the degree of suitability of the solution is reduced. Therefore, it is also necessary to demand products with guarantees and prescriptions that ensure and offer sufficient technical safety. 


\section{NOMENCLATURA}

\begin{tabular}{|c|c|}
\hline EVC & Estudio de viabilidad constructiva \\
\hline BT & Bloque de tierra \\
\hline BTC & Bloque de tierra comprimido \\
\hline BTE & Bloque de tierra extruido \\
\hline$C-C A$ & Referencia a la calidad del producto \\
\hline$C-R C$ & Referencia a los requisitos constructivos \\
\hline$C-A E$ & Referencia a las acciones externas \\
\hline EVC & Estudio de viabilidad constructiva \\
\hline $\mathrm{Gl}_{i}$ & Grado de idoneidad para los i ámbitos \\
\hline$N E T_{q}$ & Nivel de evaluación técnica de los q indicadores \\
\hline$N E T P_{i}$ & $\begin{array}{l}\text { Nivel de evaluación técnica ponderado para los } \\
\qquad i \text { ámbitos }\end{array}$ \\
\hline$W_{i}$ & Coeficiente de ponderación para los i ámbitos \\
\hline
\end{tabular}

\section{INTRODUCCIÓN}

Durante las dos últimas décadas la situación medioambiental y económica a nivel mundial está generando la necesidad y el interés por el desarrollo de soluciones constructivas adecuadas a las demandas ambientales, energéticas y sociales. En este contexto, el uso del adobe y el bloque de tierra (BT), que incluye fundamentalmente el bloque de tierra comprimida (BTC), como mampuestos de fábrica, pueden ser una alternativa más sostenible en la construcción.

Como sustento de esta afirmación, el marco actual de investigación del BT se ha centrado en estudios sobre sus propiedades mecánicas (Gandia, Gomes, Corrêa, Rodrigues y Mendes, 2019; Mahmood, Habeeb y AlJumaili, 2019), térmicas (Mosquera, Canas, Cid-Falceto y Marcos, 2014; Molar-Orozco, Velázquez-Lozano y VázquezJimánez, 2020; Miloudi et al, 2019; Wati, Bidoung, Damfeu, y Meukam, 2020) y de durabilidad (Fernandes, Peixoto, Mateus y Gervásio, 2019; Lavie Arsène, Frédéric y Nathalie, 2020; Jové Sandoval, Muñoz de la Calle y Pahíno Rodríguez, 2011). Otros avalan la utilización del BT argumentando aspectos económicos, baja toxicidad e, incluso, como producto que favorece la calidad del aire interior (Fernandes et al., 2019). Además, emplea materiales locales naturales, lo que exime en gran medida el impacto ambiental atribuido al transporte (Deboucha y Hashim, 2011).

Desde el punto de vista de aplicación del producto, es necesario que el BT alcance un mejor nivel de aceptación, similar al de otros materiales de construcción, y que se superen ciertos factores que influyen negativamente en la toma de decisiones de los técnicos: el coste de producción, la baja disponibilidad de datos técnicos del producto para la justificación de las exigencias normativas, sumado a la mala praxis por desconocimiento de las condiciones de aplicación del material. En consecuencia, es necesario establecer un marco que defina mejor las determinaciones constructivas, económicas o medioambientales del uso del BT. Éstas deben servir de sustento para que su elección sea viable y garantizada con datos técnicos y así crezca la confianza de todos los agentes que intervienen en la construcción, con el propósito de establecer directrices de uso correcto y acorde con las exigencias técnico-constructivas.

La viabilidad del uso de BTC y del adobe ha sido analizada por Maldonado Ramos, Castilla Pascual, Vela Cossío y Rivera Gómez (2001), demostrando que, para una obra de pequeñamediana escala, supone una solución económica, así como una mejora respecto al aislamiento térmico frente a otros materiales como hormigón, ladrillo o acero. Asimismo, en el ámbito normativo internacional existen diversos documentos que regulan el uso y aplicación del BT, como por ejemplo las normas brasileñas (1986-1996), colombiana (2004), peruana (2000) o la española (2008), todos ellos tratados y analizados por Cid-Falceto, Mazarrón y Cañas (2011). No obstante, ninguna de las aportaciones comentadas ofrece una herramienta que permita analizar la vialidad de aplicación del BT en edificación, por lo que su aplicabilidad es reducida al estar sometido a un criterio técnico sin suficiente ni adecuado conocimiento respecto de sus cualidades y prestaciones.

Respecto a las metodologías de evaluación, aquellas que emplean indicadores cuantitativos o cualitativos han sido extensamente desarrolladas en la literatura. En cuanto a las enfocadas en la construcción con tierra, destaca la aportación de Canivell para la evaluación de fábricas de tapia (Canivell, Rodríguez-García, González-Serrano y Romero Girón, 2020; López-Zambrano, Canivell y Calama, 2019). Aunque su cometido se centra en la evaluación del riesgo físico, ciertas operatividades de los indicadores han sido tomadas como referencia. Sin embargo, no se han desarrollado herramientas metodológicas que sirvan para evaluar la idoneidad de ciertos productos de la construcción como el BT.

Este trabajo se centra en los aspectos constructivos que repercuten en la idoneidad del BT como producto, para lo que se han definido sus características físicas, químicas y mecánicas, como también las fases de producción y las técnicas de construcción, en aras de adoptar soluciones adaptadas a diferentes contextos. En este caso, el marco de exigencias para analizar la viabilidad del BT es la norma española de edificación (España, 2008). Los objetivos de este artículo son (I) establecer las determinaciones constructivas del BT y sus indicadores asociados, (II) exponer y validar el procedimiento metodológico de una herramienta para evaluar los aspectos constructivos de un diseño arquitectónico a nivel de proyecto básico desarrollado mediante BT, (III) exponer los resultados de dicha herramienta en los casos de estudio considerados, y (IV) analizar la respuesta de los indicadores empleados. Se estima que esta labor, es decir, establecer claramente las exigencias y determinaciones de esta herramienta, ofreciendo un análisis de indicadores, facilitará la toma de decisiones en este ámbito y, consecuentemente, contribuirá a optimizar la aplicabilidad del BT como solución constructiva sostenible. 


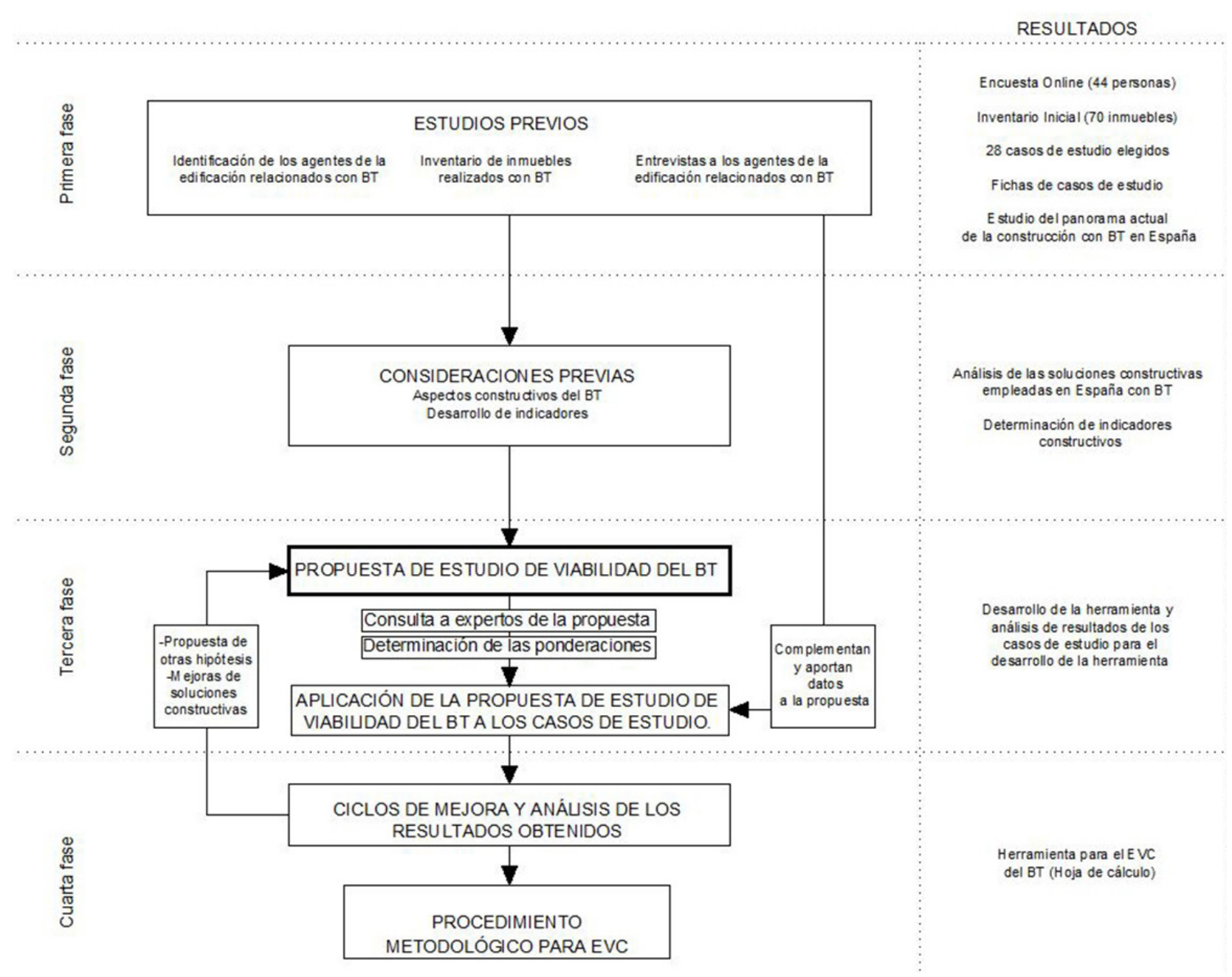

Figura 1. Procedimiento metodológico que se establece para establecer el EVC. Fuente: Elaboración de los autores.

\section{PROCEDIMIENTO METODOLÓGICO DE LA INVESTIGACIÓN. INDICADORES}

El proceso metodológico llevado a cabo para plantear la herramienta, que se denominará estudio de viabilidad constructiva (EVC), se ha compartimentado en las fases y contenidos que se detallan en la Figura 1.

A partir del inventario realizado sobre edificaciones que han empleado en su diseño BT en España, se establece, en una primera fase, un conjunto de 70 casos analizados que corresponden a 59 viviendas (uso residencial) y a otros 11 inmuebles de usos terciarios, docentes o industriales. Posteriormente, con en el trabajo de campo se seleccionan los casos más relevantes, quedando un total de 28 de los 70 estudiados, que se estructuran en 15 casos de uso residencial unifamiliar, 5 de uso plurifamiliar, 6 de uso terciario y 2 edificios docentes. En la segunda fase, se catalogan las determinaciones constructivas asociadas al BT (Tabla 1). En la tercera, se implementa la herramienta en los 28 casos citados y mediante ciclos de mejora, se adecúa su respuesta óptima. En la última fase, se valida el funcionamiento del EVC en un caso de estudio no incluido en los 28 previos.

La primera clave del EVC consiste en plantear las determinaciones constructivas (segunda fase) que se organizan, atendiendo a las tres categorías desarrolladas en la Tabla 1: las características del producto (calidad), las exigencias constructivas (requisitos del propio sistema constructivo) y los condicionantes externos (acciones externas). Así, la calidad del producto contempla las características físicas, químicas y mecánicas del BT, definidas y/o declaradas por el fabricante y establecidas en el proyecto. En segundo lugar, los requisitos constructivos se asocian al producto para dar respuesta a ciertos aspectos del cumplimiento de la normativa vigente (Ministerio de Fomento, 1999), fundamentalmente la estabilidad estructural de la fábrica y la habitabilidad de los espacios. Y, respecto a la tercera categoría, habrá que considerar el análisis de las acciones externas que pueden afectar a la fábrica de BT a lo largo de su vida útil, las que dependen de la función que cumpla el muro (soporte de carga o cerramiento), de la localización y orientación de este, y de la agresividad del ambiente al que se expone (Soronis, 1992). 
INDICADORES: Calidad del producto (C-CA)

DETERMINACIONES: Calidad del producto

\begin{tabular}{|c|c|c|c|c|}
\hline & & -001 & Densidad & $\begin{array}{c}\text { Densidad aparente y seca para } \\
\text { exigencias acústicas }\end{array}$ \\
\hline & & $\begin{array}{l}\text { Resistencia } \\
\text { mecánica }\end{array}$ & $\begin{array}{l}\text { Resistencia a compresión simple } \\
\text { declarada (UNE 41410) }\end{array}$ & \\
\hline & & $\begin{array}{c}\text { Resistencia } \\
\text { a ciclos de } \\
\text { humectación/ } \\
\text { secado }\end{array}$ & $\begin{array}{c}\text { Resistencia a los ciclos en condiciones } \\
\text { severas al exterior }\end{array}$ & \\
\hline Aspec & $\begin{array}{l}\text { s físicos } \\
2\end{array}$ & $\begin{array}{l}\text { Resistencia a la } \\
\text { erosión }\end{array}$ & $\begin{array}{l}\text { Resistencia a los ciclos en condiciones } \\
\text { severas al exterior }\end{array}$ & \\
\hline & 4 & $\begin{array}{l}\text { Absorción } \\
\text { de agua por } \\
\text { capilaridad }\end{array}$ & $\begin{array}{c}\text { Resistencia a los ciclos en condiciones } \\
\text { severas al exterior }\end{array}$ & \\
\hline & 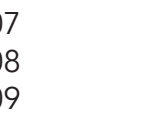 & $\begin{array}{l}\text { Resistencia a } \\
\text { ciclos de hielo/ } \\
\text { deshielo }\end{array}$ & $\begin{array}{c}\text { Resistencia evaluada y/o declarada en } \\
\text { zonas de riesgo }\end{array}$ & \\
\hline & & $\begin{array}{c}\text { Propiedades } \\
\text { térmicas del } \\
\text { producto }\end{array}$ & $\begin{array}{l}\text { Caracterización de la conductividad } \\
\text { térmica y del calor específico }\end{array}$ & \\
\hline & & $\begin{array}{l}\text { Permeabilidad al } \\
\text { vapor de agua }\end{array}$ & $\begin{array}{l}\text { Caracterización de la permeabilidad- } \\
\text { resistencia al vapor de agua }\end{array}$ & \\
\hline & & $\begin{array}{l}\text { Adherencia a } \\
\text { cortante }\end{array}$ & $\begin{array}{l}\text { Resistencia a cortante (fábricas } \\
\text { portantes) }\end{array}$ & \\
\hline & & -001 & $\begin{array}{c}\text { Caracterización química: tierra y } \\
\text { adiciones }\end{array}$ & $\begin{array}{l}\text { Estabilizantes químicos: cemento, cal, } \\
\text { yesos, silicatos }\end{array}$ \\
\hline Aspect & químicos & El agua & $\begin{array}{c}\text { Agua: composición, contenidos de sales } \\
\text { y orgánicos }\end{array}$ & \\
\hline & & $\begin{array}{l}\text { Reacción al } \\
\text { fuego }\end{array}$ & Reacción al fuego de la pieza & \\
\hline $\begin{array}{r}\text { C- } \\
\text { Aspectos }\end{array}$ & $\begin{array}{l}\text {-AG } \\
\text { eométricos }\end{array}$ & -001 & Dimensiones y tolerancias & $\begin{array}{c}\text { Descripción del tipo, dimensiones, } \\
\text { aparejos y tolerancias }\end{array}$ \\
\hline & & Aspecto & Planeidad, defectos y alveolos & \\
\hline & INDIC & DORES: Requisitos & constructivos (C-RC) & $\begin{array}{l}\text { DETERMINACIONES: Requisitos } \\
\text { constructivos }\end{array}$ \\
\hline & & -001.1 & Transmisión de las cargas & \\
\hline & & -001.2 & Transmisión al terreno & mortero de rejuntado, tipo de fibra, esbeltez \\
\hline & Estructural & -001.3 & Capacidad portante & y distribucion de cargas \\
\hline C-RC-S & & -001.4 & Configuración espacial & Simetrías y rigideces del muro y encuentros \\
\hline segurnada & & -001.5 & Configuración de los huecos & Distribución y tamaño de huecos \\
\hline & Incendios & -002 & Seguridad en caso de incendios & $\begin{array}{c}\text { Evaluación y/o declaración de la reacción al } \\
\text { fuego }\end{array}$ \\
\hline & Lesiones & -003 & Estado de las lesiones existentes & \\
\hline & & -001 & Higiene y salubridad & $\begin{array}{c}\text { Succión, absorción, porosidad abierta o } \\
\text { difusividad del vapor de agua }\end{array}$ \\
\hline $\mathrm{Hab}$ & $\begin{array}{l}\text { ilidad } \\
2\end{array}$ & $\begin{array}{l}\text { Aislamiento } \\
\text { acústico }\end{array}$ & $\begin{array}{l}\text { Índice de reducción acústica o valor de } \\
\text { aislamiento a ruido aéreo }\end{array}$ & \\
\hline & & $\begin{array}{l}\text { Comportamiento } \\
\text { térmico }\end{array}$ & $\begin{array}{l}\text { Evaluación de la resistencia térmica del } \\
\text { muro }\end{array}$ & \\
\hline
\end{tabular}




\begin{tabular}{|c|c|c|c|c|}
\hline \multicolumn{2}{|c|}{ INDICADORES: Acciones externas (C-AE) } & DETERMINACIONES: Acciones \\
externas
\end{tabular}

Tabla 1. Determinaciones consideradas e indicadores asociados. Fuente: Elaboración de los autores.

Las determinaciones presentadas en la Tabla 1 sirven para establecer un total de 35 indicadores asociados. Los indicadores se identifican mediante un código (CCA: indicadores constructivos de calidad; C-RC: de requisitos constructivos; $\mathrm{C}-\mathrm{AE}$ : de acciones externas) y se evalúan mediante valores numéricos basados en conceptos y apreciaciones. La valoración cuantitativa y/o cualitativa de cada indicador se denomina Nivel de Evaluación Técnica (NET) y requiere su definición básica según: (I) una descripción de sus tres posibles niveles $(1,2$ o 3), y (II) las referencias y fuentes empleadas, como sugiere la UNE 21929-1:2010 (AENOR, 2009), para definir indicadores de sostenibilidad para edificios. Los tres niveles de los indicadores se expresan como: 1 (nivel de evaluación bajo, valoración negativa), 2 (nivel medio, valoración moderada) y 3 (nivel alto, valoración positiva) (Figura 2).

\section{PROPUESTA METODOLÓGICA PARA EL EVC}

El EVC se diseña para ser aplicado en la primera etapa de elaboración del proyecto arquitectónico. En esta fase el objetivo es contemplar posibles estrategias en el diseño constructivo del muro no portante de cerramiento. El procedimiento de evaluación (Figura 3) consta de tres etapas diferenciadas: entrada de datos, establecimiento de niveles de los indicadores y evaluación que, a su vez, se desarrolla en dos etapas encadenadas.

En la primera etapa, consideradas las fuentes de información, se recopilan y clasifican las determinaciones

\begin{tabular}{|c|c|c|}
\hline \multicolumn{3}{|c|}{ Concepto: Resistencia a ciclos de humectación/secado } \\
\hline \multicolumn{3}{|c|}{ Raíz: Calidad del producto $>$ Aspectos físicos } \\
\hline \multicolumn{3}{|c|}{$\begin{array}{l}\text { Descripción del parámetro: Conocer con antelación la resistencia a los ciclos de humectación } \\
\text { y secado del BT aportará datos acerca de posibles soluciones constructivas que no supongan un } \\
\text { deterioro del material ante exposiciones severas. }\end{array}$} \\
\hline \multicolumn{3}{|c|}{$\begin{array}{l}\text { Consideraciones técnicas: } \\
\text { ¿Qué nos interesa saber sobre la resistencia a ciclos de humectación y secado? } \\
\text { - En el ensayo, al encontrarse en exposición severa, la fábrica debe ser capaz de } \\
\text { soportar los seis ciclos de humectación y secado sin manifestar una serie de } \\
\text { condiciones (ensayo específico de la UNE } 41410: 2008 \text { ). } \\
\text { El deterioro provocado por estos ciclos en la superficie hace que aumente la } \\
\text { descomposición del material más rápidamente (Falceto, 2012). }\end{array}$} \\
\hline \multicolumn{3}{|c|}{ Niveles: } \\
\hline Color & Nivel & Explicación \\
\hline Bajo & 1 & $\begin{array}{l}\text { En cuanto a la producción externa, el fabricante no declara ningún } \\
\text { aspecto sobre la resistencia a ciclos de humectación y secado. } \\
\text { En la producción in situ no será posible la declaración de la resistencia } \\
\text { a ciclos de humectación y secado. }\end{array}$ \\
\hline Medio & 2 & $\begin{array}{l}\text { En cuanto a la producción externa, el fabricante declara acerca de } \\
\text { la resistencia a ciclos de humectación y secado sin certificación. } \\
\text { En la producción in situ, será posible la declaración de la resistencia a } \\
\text { ciclos de humectación y secado pero no, su certificación. }\end{array}$ \\
\hline Alto & 3 & $\begin{array}{l}\text { En cuanto a la producción externa, el fabricante declara y certifica } \\
\text { la resistencia a ciclos de humectación y secado. } \\
\text { En la producción in situ, será posible la declaración y la certificación del } \\
\text { producto. }\end{array}$ \\
\hline \multicolumn{3}{|c|}{$\begin{array}{l}\text { Normativa: } \\
\text { Página } 14 \text { - UNE 41410:2008 - (Aenor, 2008b) }\end{array}$} \\
\hline \multicolumn{3}{|c|}{$\begin{array}{l}\text { Referencias: } \\
\text { (Guettala, Abibsi, \& Houari, 2006) }\end{array}$} \\
\hline
\end{tabular}

Figura 2. Definición básica del NET para el indicador de Resistencia a ciclos de humectación / secado. Fuente: Elaboración de los autores en base a normas UNE 41410 (2008) y ASTM D559 (1989). 


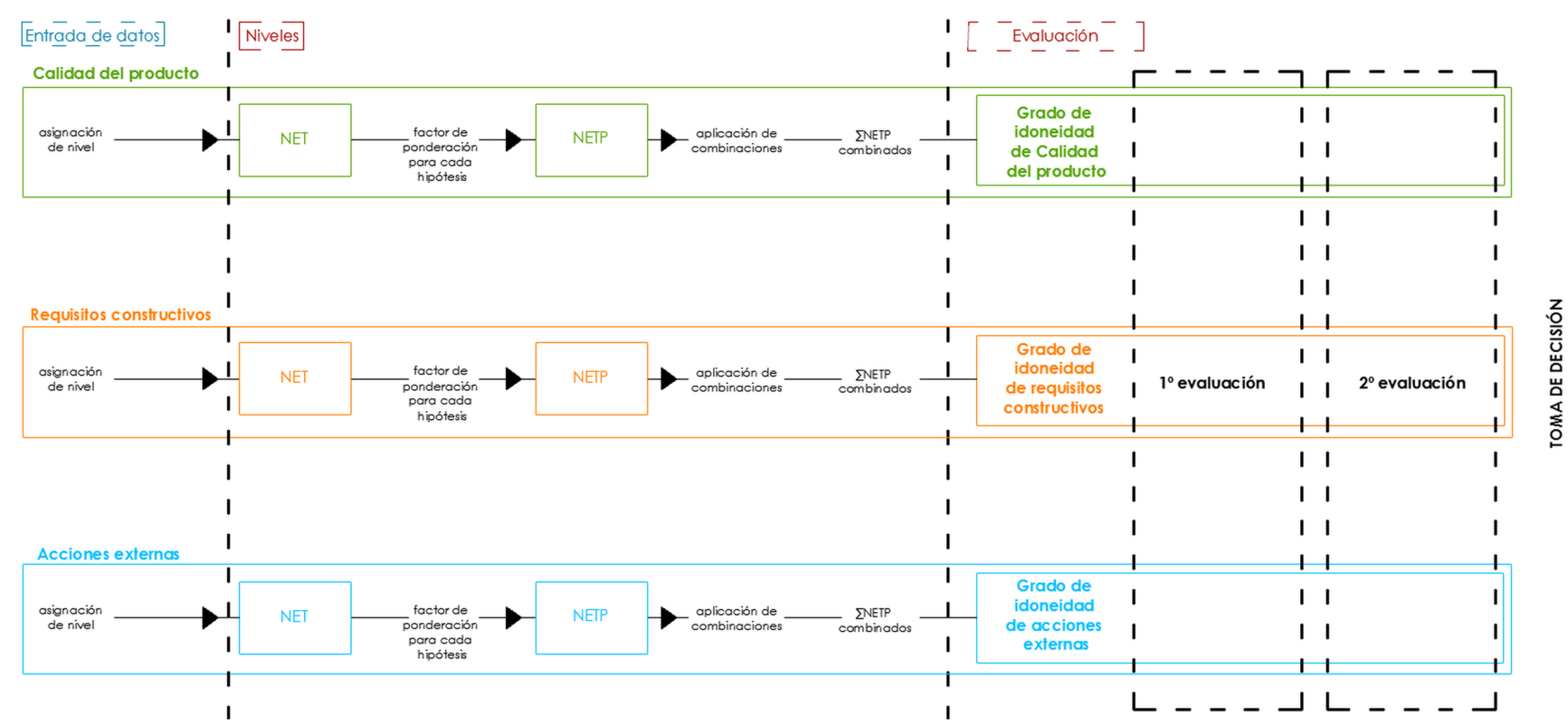

Figura 3. Etapas del procedimiento de la EVC. Fuente: Elaboración de los autores.

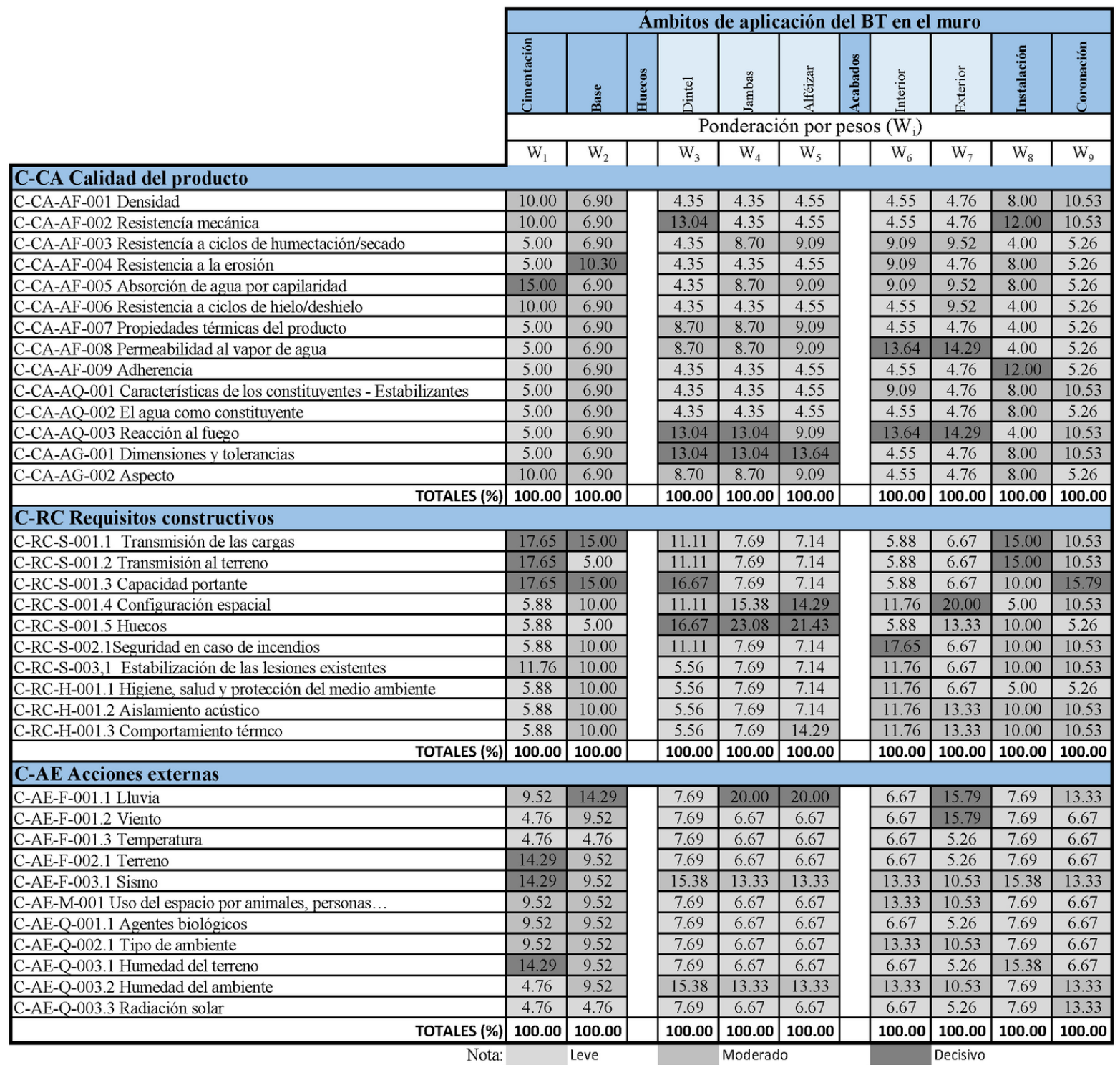

Figura 4. Resultado de la encuesta a expertos para establecer los promedios de pesos (Wi) en cada ámbito del muro de todos los indicadores. Fuente: Elaboración de los autores. 


\begin{tabular}{|c|c|c|c|c|c|c|c|c|c|c|c|c|}
\hline \multirow{3}{*}{ Combinaciones (j) } & & & & \multicolumn{9}{|c|}{ Coeficiente K* para los ámbitos (i) } \\
\hline & \multicolumn{3}{|c|}{ Indicadores con minoración de valoración por combinación } & $\begin{array}{l}\vdots \\
0 \\
\vdots \\
0\end{array}$ & $\begin{array}{l}0 \\
0 \\
0 \\
0\end{array}$ & $\begin{array}{l}- \\
0 \\
0 \\
0 \\
0 \\
0\end{array}$ & $\begin{array}{l}\Omega \\
0 \\
0 \\
0 \\
0 \\
0\end{array}$ & $\begin{array}{c}0 \\
0 \\
0 \\
0 \\
0 \\
1 \\
0\end{array}$ & $\begin{array}{c}\overline{0} \\
1 \\
0 \\
0 \\
1 \\
0\end{array}$ & $\begin{array}{l}1 \\
0 \\
1 \\
0 \\
1 \\
0\end{array}$ & $\begin{array}{l} \\
1 \\
0 \\
0 \\
0 \\
0\end{array}$ & 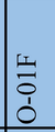 \\
\hline & Calidad (CA) & Requisitos Constructivos (RC) & Acciones externas (AE) & 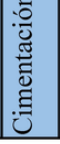 & 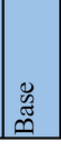 & $\begin{array}{l}\bar{\Xi} \\
\stackrel{\Xi}{\Xi} \\
\end{array}$ & $\begin{array}{l}\text { D } \\
\text { हే } \\
\text { E్ } \\
\text {. }\end{array}$ & 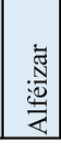 & 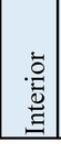 & 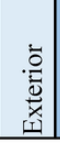 & 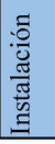 & 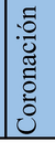 \\
\hline $\mathrm{N}^{\circ} 01$ & & & C-AE-F-001.1/ C-AE-F-001.2 & 0 & 0.5 & 0.5 & 0.5 & 0.5 & 0 & 0.5 & 0.5 & 0.5 \\
\hline $\mathrm{N}^{\circ} 02$ & & & $\begin{array}{l}\text { C-AE-F-001.1/ C-AE-F-002.1/ } \\
\text { C-AE-Q-003.1/ C-AE-M-001 }\end{array}$ & 0.5 & 0.5 & 0 & 0 & 0 & 0 & 0 & 0 & 0 \\
\hline $\mathrm{N}^{\mathrm{o}} 03$ & & & C-AE-Q-001.1 & 0.5 & 0.5 & 0.5 & 0.5 & 0.5 & 0.5 & 0.5 & 0 & 0.5 \\
\hline $\mathrm{N}^{\mathrm{o}} 04$ & & C-RC-S-001.4 & C-AE-F-002.1/ C-AE-F-003.1 & 0 & 0 & 0.5 & 0.5 & 0 & 0 & 0 & 0 & 0.5 \\
\hline $\mathrm{N}^{\mathrm{o}} 05$ & & C-RC-ES-001 & C-AE-Q-003.2 & 0 & 0 & 0.5 & 0.5 & 0.5 & 0.5 & 0.5 & 0 & 0 \\
\hline $\mathrm{N}^{\mathrm{o}} 06$ & $\begin{array}{l}\text { C-CA-AF-005/ } \\
\text { C-CA-AF-008 }\end{array}$ & C-RC-ES-001 & C-AE-F-001.3/ C-AE-Q-003.2 & 0 & 0.5 & 0.5 & 0.5 & 0.5 & 0 & 0 & 0 & 0.5 \\
\hline $\mathrm{N}^{\circ} 07$ & & $\begin{array}{c}\text { C-RC-S-001.1/ C-RC-S-001.4/ } \\
\text { C-RC-S-001.5 }\end{array}$ & & 0 & 0.5 & 0.5 & 0 & 0 & 0 & 0 & 0 & 0.5 \\
\hline
\end{tabular}

Figura 5. Combinaciones críticas según la encuesta a expertos y sus coeficientes de Kj de ponderación según los ámbitos establecidos. Fuente: Elaboración de los autores.

constructivas del caso de estudio conforme a las categorías de la Tabla 1, lo que sirve para valorar posteriormente los indicadores asociados, según el NET para cada uno de los 35 indicadores de las tres categorías. Cabe aclarar que al ser cada indicador de diferente naturaleza y distintos los ámbitos de aplicación dentro de un muro, no todos los indicadores van a tener el mismo grado de influencia en la evaluación, debido a lo cual sus valores no deben sumarse directamente. Por ello, se establecen métodos de ponderación, según la UNE-ISO/TS 21929 (AENOR, 2009), mediante la aplicación de pesos o coeficientes de corrección elaborados a partir de encuestas a expertos (ver Figura 1, fase 3), enfatizando en la valoración del grado de determinación de cada indicador, según nueve ámbitos de aplicación del BT definidos como elementos del cerramiento (se consideran: cimentación, base de muro, partes de un hueco -dintel, jambas, alfeizar-, acabados -interior y exterior- instalaciones y coronación de muro) (Figura 4). En dichas encuestas también se incluyen tres tipos de indicadores, determinados según los pesos asociados: decisivo, moderado o leve. Los rangos del NET (1 a 3) se ponderan en función de la relación entre los indicadores propuestos y los nueve ámbitos definidos del muro. Así, los NET disminuirán o mantendrán su valor de forma proporcional mediante el producto con el coeficiente, obteniendo el nivel de evaluación técnica ponderada (NETP).

Por consiguiente, al existir nueve ámbitos, tal como refleja la Figura 4, se obtienen nueve conjuntos de NETP, luego de aplicar la siguiente ecuación [1]:

$$
N E T P_{i}=\frac{N E T \times W_{i}}{100}[1]
$$

siendo $W_{i}$ el peso para cada uno de los nueve ámbitos estudiados.

Las encuestas a expertos también sirven para considerar cuáles son las circunstancias más adversas cuando existan ciertas combinaciones críticas. A partir de ellas se establecen, entonces, siete combinaciones críticas de indicadores que minoran las valoraciones de los NETPi. De esta forma, para cada uno de los nueve ámbitos se desarrollarían algunas de las siete combinaciones posibles que se detallan en la Figura 5. Al establecer la condición de que para NET $<3$ de los indicadores asociados a las combinaciones, las valoraciones de sus correspondientes NETP se reducirán un 50\% mediante el coeficiente $K_{i}$, quedando la ponderación de NET según la ecuación [2].

$$
N E T P_{i, j, q}=\frac{N E T_{q} \times W_{i}}{100} \times K_{j}[2]
$$

A continuación, se suman todos los NETP en cada bloque (C-CA, C-RC, C-AE) y por combinación, dentro de cada uno de los nueve ámbitos del muro, tal como se detalla en el esquema de la Figura 6. El grado de idoneidad de cada ámbito (en adelante $\mathrm{Gl}$ ) correspondería al mínimo de las combinaciones realizadas, obteniéndose con el promedio de los nueve $G I_{i}$ el Gl por cada bloque (C-CA, C-RC, C-AE), con el que se desarrollarán los dos niveles de evaluación. 


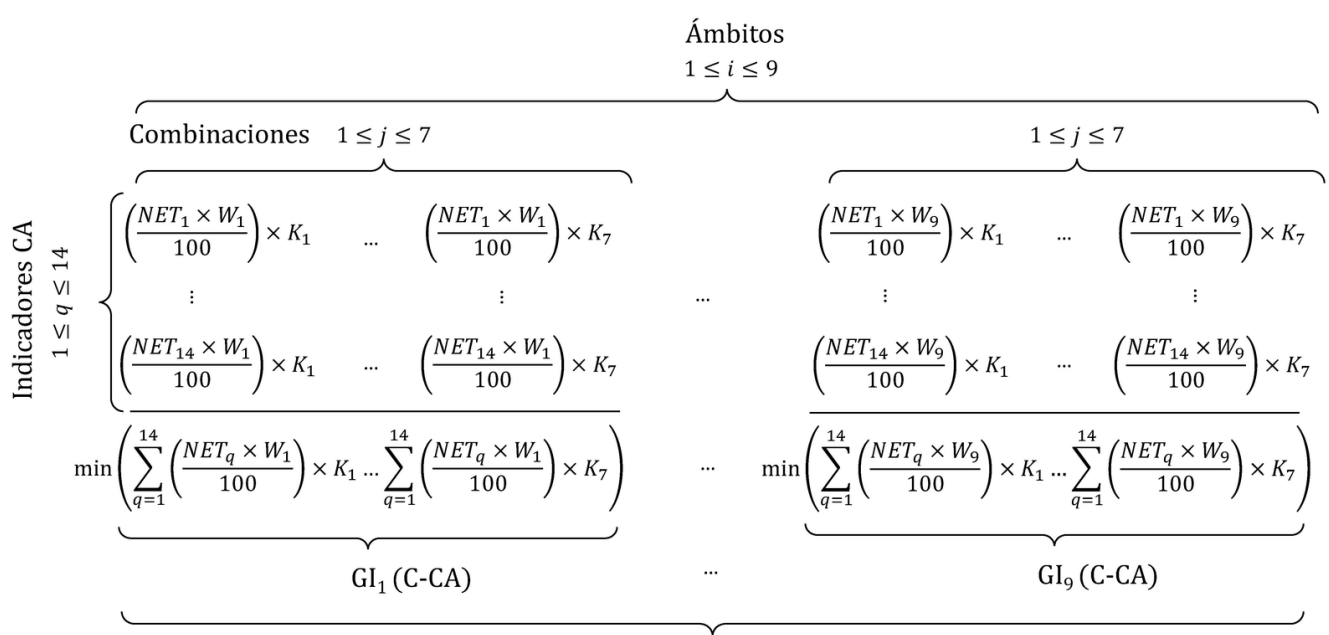

Promedio

Grado Idoneidad, GI (C-CA)

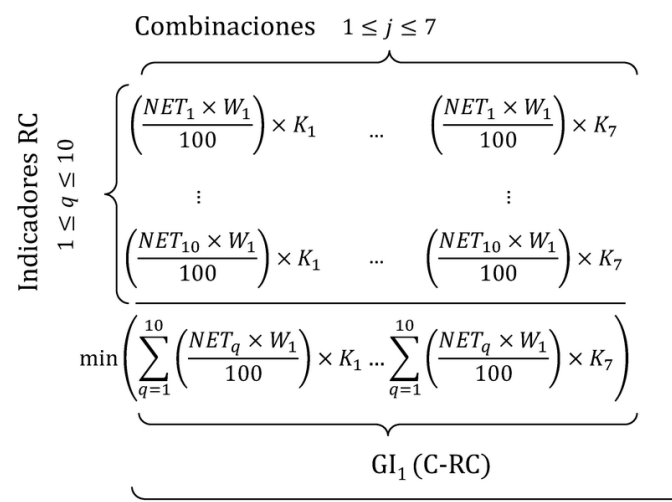

$\overbrace{}^{1 \leq j \leq 7}$

$\left(\frac{N E T_{1} \times W_{9}}{100}\right) \times K_{1} \quad \ldots \quad\left(\frac{N E T_{1} \times W_{9}}{100}\right) \times K_{7}$

: $\quad$ :

$\left(\frac{N E T_{10} \times W_{9}}{100}\right) \times K_{1} \quad \cdots \quad\left(\frac{N E T_{10} \times W_{9}}{100}\right) \times K_{7}$

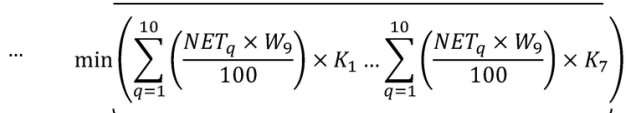

$\mathrm{GI}_{9}(\mathrm{C}-\mathrm{RC})$

Promedio

Grado Idoneidad, GI (C-RC)

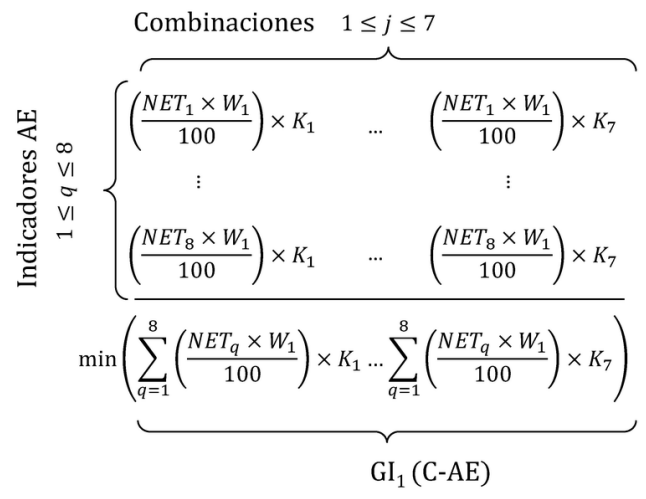

$$
\begin{aligned}
& 1 \leq j \leq 7
\end{aligned}
$$

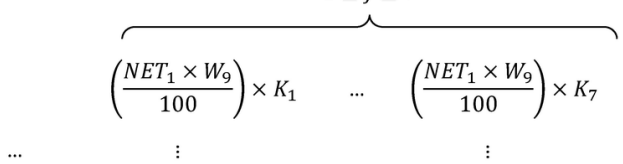

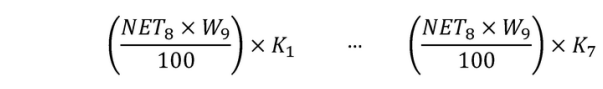

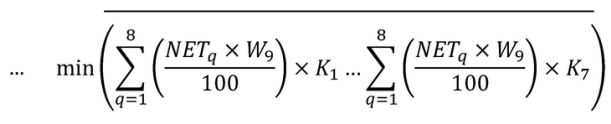

$$
\begin{aligned}
& \ldots \quad \mathrm{GI}_{9}(\mathrm{C}-\mathrm{AE})
\end{aligned}
$$

Promedio

Grado Idoneidad, GI (C-AE)

Figura 6. Secuencia de etapas en la evaluación del NET. Fuente: Elaboración de los autores.

El primer nivel de evaluación del Gl permite obtener una respuesta afirmativa ("Adecuado") o negativa ("No adecuado") sobre la viabilidad constructiva del BT. Para ello, se establece un criterio final dependiente de los Gl, evaluado como "Adecuado" siempre que los GI promedio de los tres bloques $(G I(C-C A)=1.5, G I(C-R C)$ $=2.3, G I(C-A E)=2.5)$ sean mayores o iguales a unos umbrales de Gl preestablecidos (consultar también en la Figura 11). La calificación de "Adecuado" implica que las soluciones constructivas son viables desde un punto de vista constructivo y podrían ser implementadas en el proyecto de ejecución. Sin embargo, la respuesta negativa de "No adecuado" supondría desarrollar el segundo nivel de evaluación.

En aquel segundo nivel de evaluación, se estudian pormenorizadamente los indicadores en base a dos vías de análisis para identificar deficiencias y proponer 


\begin{tabular}{|c|c|c|c|c|c|c|c|}
\hline \multirow{2}{*}{ Ámbito } & \multirow{2}{*}{ Bloque $\left({ }^{*}\right)$} & \multicolumn{2}{|c|}{ Gl bajo } & \multicolumn{2}{c|}{ GI moderado } & \multicolumn{2}{c|}{ Gl elevado } \\
\cline { 2 - 8 } & & Min & Máx & Min & Máx & Min & Máx \\
\hline \multirow{2}{*}{ Cimentación } & $\mathrm{RC}$ & 0 & 2.32 & 2.33 & 2.69 & 2.7 & 3 \\
\cline { 2 - 8 } & $\mathrm{AE}$ & 0 & 2.32 & 2.33 & 2.69 & 2.7 & 3 \\
\hline \multirow{2}{*}{ Base del muro } & $\mathrm{RC}$ & 0 & 2.29 & 2.3 & 2.53 & 2.54 & 3 \\
\cline { 2 - 8 } & $\mathrm{AE}$ & 0 & 2.1 & 2.11 & 2.59 & 2.6 & 3 \\
\hline \multirow{2}{*}{ Huecos - Dintel } & $\mathrm{RC}$ & 0 & 2.18 & 2.19 & 2.59 & 2.6 & 3 \\
\cline { 2 - 8 } & $\mathrm{AE}$ & 0 & 1.99 & 2 & 2.59 & 2.6 & 3 \\
\hline \multirow{2}{*}{ Huecos -Jambas } & $\mathrm{RC}$ & 0 & 2.18 & 2.19 & 2.58 & 2.59 & 3 \\
\cline { 2 - 8 } & $\mathrm{AE}$ & 0 & 1.99 & 2 & 2.59 & 2.6 & 3 \\
\hline \multirow{2}{*}{ Huecos -Alféizar } & $\mathrm{RC}$ & 0 & 2.24 & 2.25 & 2.58 & 2.59 & 3 \\
\cline { 2 - 8 } & $\mathrm{AE}$ & 0 & 1.98 & 1.99 & 2.59 & 2.6 & 3 \\
\hline \multirow{2}{*}{ Acabados - Interior } & $\mathrm{RC}$ & 0 & 2.25 & 2.26 & 2.45 & 2.46 & 3 \\
\cline { 2 - 8 } & $\mathrm{AE}$ & 0 & 1.99 & 2 & 2.58 & 2.59 & 3 \\
\hline \multirow{2}{*}{ Acabados- Exterior } & $\mathrm{RC}$ & 0 & 2.32 & 2.33 & 2.58 & 2.59 & 3 \\
\cline { 2 - 8 } & $\mathrm{AE}$ & 0 & 2.19 & 2.2 & 2.58 & 2.59 & 3 \\
\hline \multirow{2}{*}{ Recursos técnicos } & $\mathrm{RC}$ & 0 & 2.29 & 2.3 & 2.7 & 2.71 & 3 \\
\cline { 2 - 8 } & $\mathrm{AE}$ & 0 & 2.29 & 2.3 & 2.7 & 2.71 & 3 \\
\hline \multirow{2}{*}{ Coronación } & $\mathrm{RC}$ & 0 & 2.39 & 2.4 & 2.59 & 2.6 & 3 \\
\cline { 2 - 8 } & $\mathrm{AE}$ & 0 & 2.18 & 2.19 & 2.58 & 2.59 & 3 \\
\hline \multirow{2}{*}{ Partición } & $\mathrm{RC}$ & 0 & 2.29 & 2.3 & 2.59 & 2.6 & 3 \\
\cline { 2 - 8 } & $\mathrm{AE}$ & 0 & 2.29 & 2.3 & 2.69 & 2.7 & 3 \\
\hline \multirow{2}{*}{$(*)$ RC: requisitos constructivos; $\mathrm{AE}:$ acciones externas } & & & & \\
\hline
\end{tabular}

Figura 7. Clasificación de Gl según intervalos establecidos para el segundo nivel de evaluación. Fuente: Elaboración de los autores.

mejoras. El primer análisis corresponde a los indicadores de calidad del producto, por lo que se cruzan los NET correspondientes con las exigencias de la normativa vigente de aplicación (fundamentalmente la UNE 41410:2008) y se detectan carencias en las especificaciones técnicas de las declaraciones del fabricante, que, a su vez, puedan subsanarse $\mathrm{o}$ al menos permitir propuestas de medidas alternativas. Para tal efecto, se han diseñado en el EVC unos algoritmos condicionales que vinculan estos indicadores con los valores de entrada de datos relacionados con la calidad del producto. El segundo análisis se centra en los $\mathrm{Gl}_{\text {i }}$ de los requisitos constructivos y en las acciones externas que afectan a todos los ámbitos del muro donde el BT es utilizado. En este caso, se han establecido tres clasificaciones de los rangos de los $\mathrm{Gl}_{\text {i }}$ según sean óptimos (verde), moderados (amarillo) o bajos (rojo). Esta clasificación se elabora a partir de los intervalos de $G I_{\text {i }}$ de los 28 casos de estudio implementados (Figura 7). Dependiendo de la clasificación de cada $G l_{i^{\prime}}$ se pueden establecer posibles soluciones para mejorar los aspectos considerados deficientes.

Una vez desarrollado el procedimiento metodológico del EVC, se efectúa una validación mediante la implementación del método en un caso de estudio no incluido en el listado de los 28 seleccionados como base para la elaboración de la herramienta.

\section{VALIDACIÓN Y RESULTADOS}

El objetivo de la validación de la herramienta propuesta es verificar que el procedimiento es apto para la evaluación constructiva de muros de BT como parte de un proyecto arquitectónico. Con dicho propósito, se comprueba si los resultados del EVC de un caso de estudio cualquiera son los estimados en función de sus GI. Para realizar la validación del EVC se selecciona un edificio de tamaño suficiente (aproximadamente $700 \mathrm{~m}^{2}$ construidos), con documentación técnica disponible, donde las soluciones constructivas son variadas y emplea BT con certificación técnica en los muros de cerramiento.

Con estas condiciones de partida, se selecciona la Escuela Infantil Municipal La Font del Rieral en Santa Eulàlia de Ronçana (Barcelona), designado como BAR-001. En un sector se emplean BTC, de dimensiones $29 \times 14,5 \times 9,5 \mathrm{~cm}$, de un fabricante que aporta fichas técnicas del producto donde se justifican las exigencias normativas, aunque sin homologación oficial. Las principales características de las soluciones constructivas de los muros aparecen en la fachada del sector sur del edificio, compuesta por un muro portante de doble hoja con fábrica de BTC (cada hoja de un espesor de $14,5 \mathrm{~cm}$ ) ancladas entre sí con llaves de acero galvanizado, con una capa de aislamiento de corcho natural $(2 \mathrm{~cm})$ y cámara de aire en su interior $(5 \mathrm{~cm})$. Hacia el interior, estos muros tienen el BTC a cara vista con un zócalo revestido de paneles contrachapados de madera hasta la altura de los alfeizares de ventanas; y hacia el exterior, se tratan con hidrofugante que se reviste con mortero de cal y cemento. Los huecos se diseñan con carpintería de madera adecuadamente impermeabilizados. El apoyo del muro portante a cimentación se lleva a cabo mediante hiladas de bloques de mortero de cemento, conectadas con barras corrugadas metálicas a la zapata corrida de hormigón armado; la lámina impermeabilizante se coloca 


\begin{tabular}{|c|c|c|}
\hline \multicolumn{3}{|c|}{ Calidad del producto } \\
\hline Producto & & BTC - Bioterre \\
\hline Producción & In situ / externa & Externa \\
\hline \multirow{7}{*}{$\begin{array}{l}\text { Tipo de BT y } \\
\text { composición del } \\
\text { bloque previsto }\end{array}$} & Tipo de estabilizante & Cemento \\
\hline & Declaración de estabilizante & Sí \\
\hline & Certificación de estabilizante & Sí \\
\hline & Densidad prevista & Sí \\
\hline & Utilización de fibras & No \\
\hline & Indicación de composición del agua & No \\
\hline & Materia orgánica o sales & No \\
\hline \multirow{14}{*}{$\begin{array}{l}\text { Declaración de } \\
\text { datos }\end{array}$} & Densidad & Sí \\
\hline & Resistencia a compresión & Sí \\
\hline & Resistencia a ciclos de humectación/secado & Sí \\
\hline & Resistencia a la erosión & Sí \\
\hline & Absorción de agua por capilaridad & Sí \\
\hline & Resistencia a ciclos de hielo /deshielo & Sí \\
\hline & Propiedades térmicas del producto & Sí \\
\hline & Permeabilidad al vapor de agua & Sí \\
\hline & Reacción al fuego & Sí \\
\hline & Dimensiones & Sí \\
\hline & Tolerancias & Sí \\
\hline & Planeidad & Sí \\
\hline & Defectos & Sí \\
\hline & Alveolos & Sí \\
\hline \multirow{9}{*}{$\begin{array}{l}\text { Certificación } \\
\text { mediante } \\
\text { ensayos } \\
\text { normalizados }\end{array}$} & Densidad & Sí \\
\hline & Resistencia a compresión & Sí \\
\hline & Resistencia a ciclos de humectación/secado & Sí \\
\hline & Resistencia a la erosión & Sí \\
\hline & Absorción de agua por capilaridad & Sí \\
\hline & Resistencia a ciclos de hielo /deshielo & Sí \\
\hline & Propiedades térmicas del producto & Sí \\
\hline & Permeabilidad al vapor de agua & Sí \\
\hline & Reacción al fuego & Sí \\
\hline \multicolumn{3}{|l|}{ Agentes externos } \\
\hline \multirow{7}{*}{$\begin{array}{l}\text { Aspectos } \\
\text { ambientales }\end{array}$} & Zona pluviométrica de promedios (CTE) & III \\
\hline & Viento dominante de la zona & Levante y poniente \\
\hline & Viento $(\mathrm{km} / \mathrm{h})$ & - \\
\hline & \begin{tabular}{|l|} 
Zona eólica \\
\end{tabular} & $\mathrm{C}$ \\
\hline & Temperatura mínima & $<0^{\circ} \mathrm{C}$ \\
\hline & Humedad del ambiente & $>70 \%$ \\
\hline & Riesgo de microorganismos & Sí \\
\hline \multirow{3}{*}{$\begin{array}{l}\text { Aspectos del } \\
\text { terreno }\end{array}$} & $\begin{array}{l}\text { Descripción del perfil del terreno con respecto al lugar de } \\
\text { intervención }\end{array}$ & Levemente inclinado \\
\hline & Altura del nivel freático & Se desconoce \\
\hline & Coeficiente ag de la zona (Sismo) & 0,09 \\
\hline \multirow{5}{*}{$\begin{array}{l}\text { Aspectos } \\
\text { patrimoniales }\end{array}$} & Catálogo & No \\
\hline & Normas específicas & No \\
\hline & Grado de protección & - \\
\hline & Estrategias de sostenibilidad & No \\
\hline & Normas constructivas especificas & No \\
\hline \multirow[t]{3}{*}{ Otros } & Tipo de vegetación colindante & $\begin{array}{c}\text { De hoja caduca en la } \\
\text { fachada sur que da sombra } \\
\text { en verano y luz, en invierno }\end{array}$ \\
\hline & Tipo de contaminación posible & Ninguno \\
\hline & Uso del espacio colindante & Patio de juegos \\
\hline
\end{tabular}

\begin{tabular}{|c|c|c|}
\hline \multicolumn{3}{|c|}{ Requisitos constructivos } \\
\hline \multirow{4}{*}{$\begin{array}{l}\text { Características } \\
\text { generales del } \\
\text { inmueble }\end{array}$} & Altura & 3 metros aprox. \\
\hline & $\mathrm{N}^{\circ}$ de plantas & 1 \\
\hline & Composición espacial & No simétrica / Curva \\
\hline & Uso del edificio & Educación \\
\hline \multirow{15}{*}{$\begin{array}{l}\text { Características } \\
\text { del muro }\end{array}$} & Localización del bloque en fachada & Exterior \\
\hline & Tipo de revestimiento del muro o cara vista & Revestimiento \\
\hline & Tipo de mortero a utilizar & Mortero de cal \\
\hline & Composición del muro & $\begin{array}{c}\text { Doble fábrica de BTC de } \\
30 \mathrm{~cm} \text { con cámara y } \\
\text { aislamiento de corcho } \\
\text { natural de } 2 \mathrm{~cm} .\end{array}$ \\
\hline & Previsión de altura del apoyo del muro & $15 \mathrm{~cm}$ \\
\hline & Tipo de apoyo del muro en la cimentación & $\begin{array}{c}\text { Muro elevado por bloque } \\
\text { de hormigón y anclado a la } \\
\text { cimentación }\end{array}$ \\
\hline & Previsión de cimentación & $\begin{array}{c}\text { Zapata corrida de hormigón } \\
\text { apoyada directamente sobre } \\
\text { el firme }\end{array}$ \\
\hline & Arriostramiento del muro & Viga de coronación \\
\hline & Esbeltez & 2,5 metros de alto \\
\hline & Volumetría simétrica o irregular & Irregular \\
\hline & Huecos & Con huecos \\
\hline & Espesor previsto & $30 \mathrm{cms}$ \\
\hline & Continuidad de los elementos & Sí \\
\hline & Previsión de juntas & Sí \\
\hline & Previsión de análisis del mortero utilizado de agarre & Sí \\
\hline \multirow{2}{*}{$\begin{array}{l}\text { Elementos de } \\
\text { protección } \\
\end{array}$} & Utilización de zócalo & No \\
\hline & Utilización de cornisa & Sí \\
\hline \multirow{4}{*}{$\begin{array}{l}\text { Características } \\
\text { constructivas } \\
\text { generales }\end{array}$} & Tipología estructural & Muros de carga \\
\hline & Sistema constructiva: portante o no portante & Portante \\
\hline & Estructura: identificación de cargas y sobrecargas 1 & Verticales \\
\hline & Estructura: identificación de cargas y sobrecargas 2 & No procede \\
\hline \multirow{7}{*}{ Exigencias } & ¿Exposición severa? & No \\
\hline & ¿Cara vista? & No/Sí \\
\hline & ¿Exigencias de aislamiento térmico? & Sí \\
\hline & ¿Parámetro exterior? & Sí \\
\hline & Exigencias acústicas & Sí \\
\hline & \begin{tabular}{|l|} 
Exigencias estructurales \\
\end{tabular} & Sí \\
\hline & Exigencias de resistencia al fuego & Sí \\
\hline Nombre & Escuela Santa Eulalia de Ronçana & BAR-001 \\
\hline \multicolumn{3}{|l|}{ Ámbitos } \\
\hline \multicolumn{2}{|l|}{ Cimentación } & No \\
\hline \multicolumn{2}{|l|}{ Base del muro } & Sí \\
\hline \multirow{3}{*}{$\begin{array}{l}\text { Huecos en el } \\
\text { muro }\end{array}$} & Dintel & Sí \\
\hline & \begin{tabular}{|l|} 
Jambas \\
\end{tabular} & Sí \\
\hline & Alféizar & Sí \\
\hline \multirow{2}{*}{ Acabados } & Interior & Sí \\
\hline & Exterior & Sí \\
\hline \multicolumn{2}{|c|}{ Instalación de recursos técnicos } & Sí \\
\hline \multicolumn{2}{|c|}{ Coronación del muro } & Sí \\
\hline
\end{tabular}

Figura 8. Entrada de datos inicial para el caso de estudio BAR-001. Fuente: Elaboración de los autores.

en el arranque del muro de BTC por encima del nivel del terreno. Una viga riostra de hormigón armado corona el muro donde apoya el envigado de madera aserrada de la cubierta. El proyecto contempla las exigencias del Código Técnico de la Edificación (España).

Al analizar la información disponible del proyecto, se realiza la entrada de datos (Figura 8), buscando obtener una respuesta óptima del EVC para el primer nivel de evaluación, dado que los parámetros de partida para los tres bloques considerados resultan favorables. Todos los indicadores tienen un NET de tres, excepto C-RC-S-001.4 (Configuración espacial), C-RC-S-0021 (Seguridad en caso de incendio) y C-AE-F-001.3 (Temperatura), que se valoran con dos; y C-CA-AQ-002 (agua como constituyente), que toma valor de 1 , al no tenerse constancia de las exigencias del agua de amasado, según UNE 41410.

El primer resultado del EVC (Figura 9) muestra los NETP y $G I_{i}$ correspondientes a los diferentes ámbitos del muro estudiado, así como el Gl promedio, que posibilita superar el primer nivel de evaluación. Se confirma así la hipótesis 


\begin{tabular}{|c|c|c|c|c|c|c|c|c|c|c|c|c|}
\hline & \multirow{2}{*}{\multicolumn{10}{|c|}{$\begin{array}{c}\text { Hipótesis inicial } \\
\end{array}$}} & \multirow{4}{*}{ 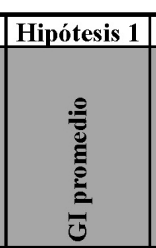 } & \multirow{4}{*}{ 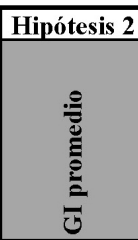 } \\
\hline & & & & & & & & & & & & \\
\hline & \multicolumn{9}{|c|}{ Ámbitos de aplicación del BT en el muro } & \multirow{2}{*}{ 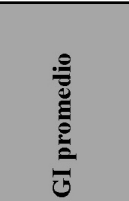 } & & \\
\hline & 总 & ڤ̆ & 离 & 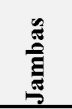 & 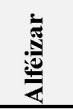 & 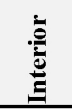 & 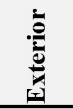 & 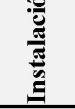 & שֶ. & & & \\
\hline Indicador & & & & & & & & & & & & \\
\hline C-CA-AF-001 Densidad & 0.00 & 0.21 & 0.13 & 0.13 & 0.14 & 0.14 & 0.14 & 0.24 & 0.32 & & & \\
\hline C-CA-AF-002 Resistencía mecánica & 0.00 & 0.21 & 0.39 & 0.13 & 0.14 & 0.14 & 0.14 & 0.36 & 0.32 & & & \\
\hline C-CA-AF-003 Resistencía a ciclos de & 0.00 & 0.21 & 0.13 & 0.26 & 0.27 & 0.27 & 0.29 & 0.12 & 0.16 & & & \\
\hline C-CA-AF-004 Resistencia a la erosión & 0.00 & 0.31 & 0.13 & 0.13 & 0.14 & 0.27 & 0.14 & 0.24 & 0.16 & & & \\
\hline C-CA-AF-005 Absorción de agua por & 0.00 & 0.21 & 0.13 & 0.26 & 0.27 & 0.27 & 0.29 & 0.24 & 0.16 & & & \\
\hline C-CA-AF-006 Resistencia a ciclos de & 0.00 & 0.21 & 0.13 & 0.13 & 0.14 & 0.14 & 0.29 & 0.12 & 0.16 & & & \\
\hline C-CA-AF-007 Propiedades térmicas del & 0.00 & 0.21 & 0.26 & 0.26 & 0.27 & 0.14 & 0.14 & 0.12 & 0.16 & & & \\
\hline C-CA-AF-008 Permeabilidad al vapor de & 0.00 & 0.21 & 0.26 & 0.26 & 0.27 & 0.41 & 0.43 & 0.12 & 0.16 & & & \\
\hline C-CA-AF-009 Adherencia & 0.00 & 0.21 & 0.13 & 0.13 & 0.14 & 0.14 & 0.14 & 0.36 & 0.16 & & & \\
\hline C-CA-AQ-001 Características de los & 0.00 & 0.21 & 0.13 & 0.13 & 0.14 & 0.27 & 0.14 & 0.24 & 0.32 & & & \\
\hline C-CA-AQ-002 El agua como constituvente & 0.00 & 0.07 & 0.04 & 0.04 & 0.05 & 0.05 & 0.05 & 0.08 & 0.05 & & & \\
\hline C-CA-AQ-003 Reacción al fuego & 0.00 & 0.21 & 0.39 & 0.39 & 0.27 & 0.41 & 0.43 & 0.12 & 0.32 & & & \\
\hline C-CA-AG-001 Dimensiones y tolerancias & 0.00 & 0.21 & 0.39 & 0.39 & 0.41 & 0.14 & 0.14 & 0.24 & 0.32 & & & \\
\hline C-CA-AG-002 Aspecto & 0.00 & 0.21 & 0.26 & 0.26 & 0.27 & 0.14 & 0.14 & 0.24 & 0.16 & & & \\
\hline $\mathrm{GI}_{\mathrm{i}}$ (C-CA) & 0.00 & 2.86 & 2.91 & 2.91 & 2.91 & 2.91 & 2.90 & 2.84 & 2.89 & 2.89 & 2.52 & 2.89 \\
\hline C-RC-S-001.1 Transmisión de las cargas & 0.00 & 0.45 & 0.33 & 0.23 & 0.21 & 0.18 & 0.20 & 0.45 & 0.32 & & & \\
\hline C-RC-S-001.2 Transmisión al terreno & 0.00 & 0.15 & 0.33 & 0.23 & 0.21 & 0.18 & 0.20 & 0.45 & 0.32 & & & \\
\hline C-RC-S-001.3 Capacidad portante & 0.00 & 0.45 & 0.50 & 0.23 & 0.21 & 0.18 & 0.20 & 0.30 & 0.47 & & & \\
\hline C-RC-S-001.4 Configuración espacial & 0.00 & 0.20 & 0.22 & 0.31 & 0.29 & 0.24 & 0.40 & 0.10 & 0.21 & & & \\
\hline C-RC-S-001.5 Configuración de los huecos & 0.00 & 0.15 & 0.50 & 0.69 & 0.64 & 0.18 & 0.40 & 0.30 & 0.16 & & & \\
\hline C-RC-S-002.1 Seguridad en caso de & 0.00 & 0.20 & 0.22 & 0.15 & 0.14 & 0.35 & 0.13 & 0.20 & 0.21 & & & \\
\hline C-RC-S-002.2 Estado de las lesiones & 0.00 & 0.30 & 0.17 & 0.23 & 0.21 & 0.35 & 0.20 & 0.30 & 0.32 & & & \\
\hline C-RC-H-001.1 Higiene, salud y protección del & 0.00 & 0.30 & 0.17 & 0.23 & 0.21 & 0.35 & 0.20 & 0.15 & 0.16 & & & \\
\hline C-RC-H-001.2 Aislamiento acústico & 0.00 & 0.30 & 0.17 & 0.23 & 0.21 & 0.35 & 0.40 & 0.30 & 0.32 & & & \\
\hline C-RC-H-001.3 Aislamiento térmico & 0.00 & 0.30 & 0.17 & 0.23 & 0.43 & 0.35 & 0.40 & 0.30 & 0.32 & & & \\
\hline $\mathrm{GI}_{\mathrm{i}}(\mathrm{C}-\mathrm{RC})$ & 0.00 & 2.80 & 2.78 & 2.77 & 2.79 & 2.71 & 2.73 & 2.85 & 2.79 & 2.78 & 2.70 & 2.20 \\
\hline C-AE-F-001.1 Pluviosidad & 0.00 & 0.43 & 0.23 & 0.60 & 0.60 & 0.20 & 0.47 & 0.23 & 0.40 & & & \\
\hline C-AE-F-001.2 Viento & 0.00 & 0.29 & 0.23 & 0.20 & 0.20 & 0.20 & 0.47 & 0.23 & 0.20 & & & \\
\hline C-AE-F-001.3 Temperatura & 0.00 & 0.10 & 0.15 & 0.13 & 0.13 & 0.13 & 0.11 & 0.15 & 0.13 & & & \\
\hline C-AE-F-002.1 Perfil del terreno & 0.00 & 0.29 & 0.23 & 0.20 & 0.20 & 0.20 & 0.16 & 0.23 & 0.20 & & & \\
\hline C-AE-F-003.1 Sismo & 0.00 & 0.29 & 0.46 & 0.40 & 0.40 & 0.40 & 0.32 & 0.46 & 0.40 & & & \\
\hline C-AE-M-001 Uso del espacio por animales, & 0.00 & 0.29 & 0.23 & 0.20 & 0.20 & 0.40 & 0.32 & 0.23 & 0.20 & & & \\
\hline C-AE-Q-001.1 Agentes biológicos & 0.00 & 0.29 & 0.23 & 0.20 & 0.20 & 0.20 & 0.16 & 0.23 & 0.20 & & & \\
\hline C-AE-Q-002.1 Actividades del hombre & 0.00 & 0.29 & 0.23 & 0.20 & 0.20 & 0.40 & 0.32 & 0.23 & 0.20 & & & \\
\hline C-AE-Q-003.1 Humedad del terreno & 0.00 & 0.29 & 0.23 & 0.20 & 0.20 & 0.20 & 0.16 & 0.46 & 0.20 & & & \\
\hline C-AE-Q-003.2 Humedad del ambiente & 0.00 & 0.29 & 0.46 & 0.40 & 0.40 & 0.40 & 0.32 & 0.23 & 0.40 & & & \\
\hline C-AE-Q-003.3 Radiación solar & 0.00 & 0.14 & 0.23 & 0.20 & 0.20 & 0.20 & 0.16 & 0.23 & 0.40 & & & \\
\hline $\mathbf{G I}_{\mathbf{i}}(\mathrm{C}-\mathrm{AE})$ & 0.00 & 2.95 & 2.92 & 2.93 & 2.93 & 2.93 & 2.95 & 2.92 & 2.93 & 2.93 & 2.93 & 2.93 \\
\hline GI (Viabilidad) & & & & & & & & & & Aceptado & Aceptado & No aceptade \\
\hline
\end{tabular}

Figura 9. Resultados de los NETP y GI (primer nivel de evaluación) del caso de estudio seleccionado. Fuente: Elaboración de los autores.

inicial, en la que se estimaba que el tipo de BT empleado era viable para las soluciones del proyecto.

En el segundo nivel de evaluación, todas las exigencias se cumplen (Figura 10) para el bloque de indicadores de calidad (C-CA). Respecto a los GI, de los bloques C-RC y $C-A E$, se determinan unos valores cercanos a 3 , por lo tanto, demuestran también una excelente viabilidad constructiva por las soluciones constructivas propuestas.

A continuación, en aras de conseguir diferentes respuestas de la herramienta, se asignan variaciones constructivas hipotéticas. Se establecen, entonces, variantes donde se analiza qué $G I$ no es adecuado y se verifica cuáles son las soluciones que se proponen. La primera hipótesis se centra en alterar la calidad del producto suponiendo que no se dispone de ciertas declaraciones técnicas: resistencia a ciclos de humectación (indicador C-CAAF-003, NET=1), resistencia a la erosión (indicador C-CA-
AF-004, NET=1) y resistencia a ciclos hielo-deshielo (indicador C-CA-AF-006, NET=1). Además, se asume que la hoja exterior del muro de BTC está sin revestir, cambiando los datos de entrada según estos mismos criterios. Consecuentemente, el resultado del segundo nivel de evaluación en cuanto a la calidad, refleja un incumplimiento en tres aspectos exigidos por UNE 41410, que garantizarían una calidad óptima al grado de exposición elevado: ensayos de absorción de agua por capilaridad, resistencia a ciclos de hielo/deshielo y permeabilidad al vapor de agua. Los GI, (C-RC) se modifican levemente al haberse alterado el indicador C-RC-H-001 que controla la respuesta higroscópica del cerramiento, ahora expuesto; mientras que los $\mathrm{Gl}_{i}$ (C$A E)$ quedan inalterados al no haberse modificado las condiciones (Figura 10). Se puede comprobar, además, que estos cambios no suponen un incumplimiento de la primera evaluación (Figura 9). 


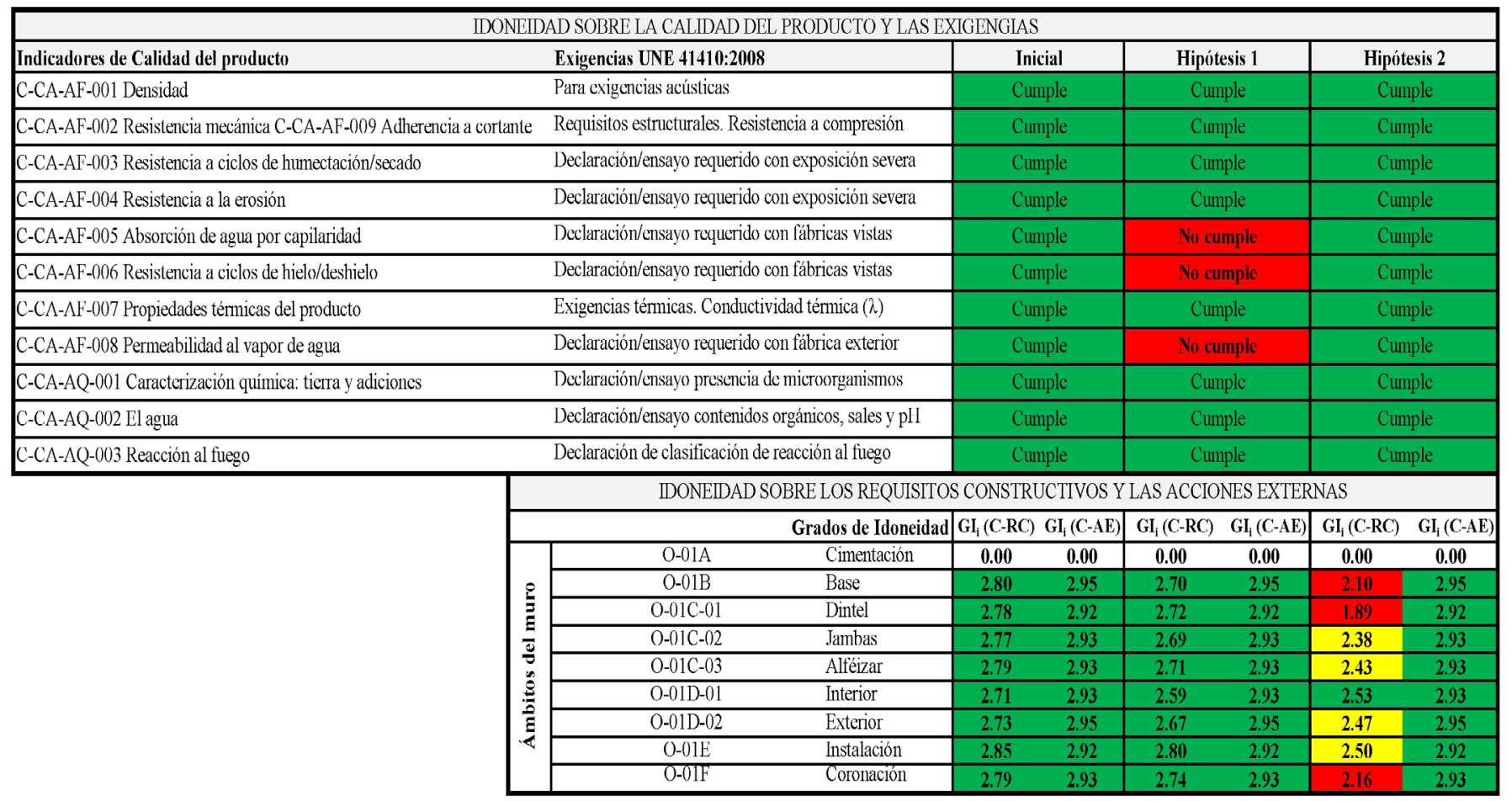

Figura 10. Resultados del segundo nivel de evaluación para el caso de estudio. Fuente: Elaboración de los autores.

En la segunda hipótesis, partiendo del estado inicial, se alteran algunas soluciones constructivas, que implican una transmisión excéntrica de las cargas sobre cimentación (C-RC-S-001.1, NET=2), una esbeltez del muro no adecuada (>1:10) y existencia de elementos que disminuyen la capacidad portante del muro (por ejemplo, mortero de relleno de juntas no adecuado) (C-RC-S-001.3, NET=2). Además, los huecos presentarían unas dimensiones mayores a las recomendadas para la construcción con tierra (Walker, 2001) (C-RC-S-001.5, NET=1). Respecto al primer nivel de evaluación (Figura 9), se observará un incumplimiento, debido a que $\mathrm{Gl}_{i}(\mathrm{C}-\mathrm{RC})$ es menor del medio establecido (2.3). Atendiendo al segundo nivel, se cumplen las exigencias de la UNE 41410, ya que la calidad del producto no se altera (Figura 10). Igualmente, como las circunstancias externas tampoco cambian, los GI. (C-AE) siguen siendo favorables. No obstante, los $G I_{i}(\mathrm{C}-\mathrm{RC})$ muestran valoraciones bajas o medias, específicamente en los ámbitos más críticos: base, coronación y dinteles, por lo que sería necesario revisar las soluciones constructivas propuestas fundamentalmente en estos ámbitos.

Finalmente, como síntesis, en la Figura 11 se reflejan los resultados obtenidos para el primer nivel de evaluación de todos los casos de estudio empleados en el diseño de esta herramienta.

Se concluye que, de los 28 casos analizados, se obtienen diversos resultados que pueden reproducir, a nivel general, ciertas pautas comunes en un proyecto de edificación. Por consiguiente, a pesar de que estos casos no son representativos estadísticamente, permiten generar una herramienta válida de retroalimentarse. El cumplimiento del primer nivel de evaluación se da en la mayoría de casos, aunque las causas de patologías son mucho más diversas y reflejan que el inconveniente reside ya sea en la calidad del producto suministrado o en las condiciones adversas del contexto (incluso en ambas simultáneamente).

\section{CONCLUSIONES}

El procedimiento metodológico de la investigación ha permitido validar el funcionamiento de la herramienta del EVC para evaluar las determinaciones de diseño de los muros de BT, ya que los resultados obtenidos son coherentes con la realidad constructiva del caso de estudio empleado. De este modo, el EVC podría ser implementado en cualquier diseño arquitectónico que use BT, lo que ayudaría a su utilización con un mejor respaldo técnico capaz de asegurar mejores resultados y favorecer el empleo de materiales de bajo impacto ambiental, como este producto.

Se insiste en que el empleo de indicadores, con una ponderación objetiva y ajustada a la realidad constructiva y al material, contribuye a que la toma de decisiones técnicas sea imparcial y objetiva, y 


\begin{tabular}{|c|c|c|c|c|}
\hline & \multicolumn{3}{|c|}{ Grados de idoneidad (GI) } & \\
\hline & C-CA Calidad del producto & C-RC Requisitos constructivos & C- AE Acciones externas & \\
\hline Valor medio GI & 1.5 & 2.3 & 2.5 & \multirow{2}{*}{$\begin{array}{c}\text { Resultado nivel } 1 \\
\text { de evaluación }\end{array}$} \\
\hline Casos de estudio & & & & \\
\hline \multicolumn{5}{|l|}{ Andalucía } \\
\hline ALM-001 & 2.31 & 2.83 & 2.80 & Adecuado \\
\hline ALM-002 & 2.52 & 2.40 & 2.49 & No Adecuado \\
\hline GRA-001 & 1.27 & 2.56 & 2.52 & No Adecuado \\
\hline MAL-001 & 1.28 & 2.11 & 1.81 & No Adecuado \\
\hline SEV-001 & 2.52 & 2.83 & 2.87 & Adecuado \\
\hline SEV-002 & 1.16 & 2.60 & 2.87 & No Adecuado \\
\hline SEV-003 & 1.17 & 2.69 & 2.50 & No Adecuado \\
\hline \multicolumn{5}{|l|}{\begin{tabular}{|l|} 
Aragón \\
\end{tabular}} \\
\hline ZAR-001 & 1.42 & 2.72 & 2.92 & No Adecuado \\
\hline ZAR-002 & 2.52 & 2.72 & 2.72 & Adecuado \\
\hline HUE-001 & 2.52 & 2.83 & 2.72 & Adecuado \\
\hline \multicolumn{5}{|l|}{ Baleares } \\
\hline BAL-001 & 3.00 & 3.00 & 3.00 & Adecuado \\
\hline \multicolumn{5}{|l|}{ Castilla y León } \\
\hline PAL-001 & 1.41 & 2.82 & 2.52 & No Adecuado \\
\hline PAL-002 & 1.43 & 2.73 & 2.93 & No Adecuado \\
\hline PAL-003 & 1.43 & 3.00 & 2.84 & No Adecuado \\
\hline \begin{tabular}{|l|} 
SEG-001 \\
\end{tabular} & 2.70 & 2.81 & 2.66 & Adecuado \\
\hline \multicolumn{5}{|l|}{ Cataluña } \\
\hline$\overline{B A R-002}$ & 3.00 & 2.80 & 2.70 & $\overline{\text { Adecuado }}$ \\
\hline \begin{tabular}{|l|} 
GER-001 \\
\end{tabular} & 3.00 & 2.93 & 2.71 & Adecuado \\
\hline GER-002 & 3.00 & 2.75 & 2.70 & Adecuado \\
\hline \multicolumn{5}{|l|}{ Madrid } \\
\hline MAD-001 & 2.44 & 2.78 & 2.63 & Adecuado \\
\hline MAD-002 & 2.45 & 2.70 & 2.55 & Adecuado \\
\hline MAD-003 & 2.45 & 2.68 & 2.34 & No Adecuado \\
\hline MAD-004 & 2.45 & 2.68 & 2.34 & No Adecuado \\
\hline MAD-005 & 2.45 & 2.62 & 2.31 & No Adecuado \\
\hline MAD-006 & 2.45 & 2.91 & 3.00 & Adecuado \\
\hline MAD-007 & 2.45 & 2.68 & 2.79 & Adecuado \\
\hline MAD-008 & 2.44 & 2.81 & 2.39 & No Adecuado \\
\hline MAD-009 & 2.52 & 2.70 & 3.00 & Adecuado \\
\hline \multicolumn{5}{|l|}{ C. Valenciana } \\
\hline ALI-001 & $\overline{3.00}$ & 3.00 & 3.00 & $\overline{\text { Adecuado }}$ \\
\hline
\end{tabular}

Figura 11. Resultados del primer nivel de evaluación del EVC para todos los casos de estudio. Fuente: Elaboración de los autores.

no se vea influenciada por prejuicios sociales o por desconocimiento con respecto al uso del BT.

La posibilidad que se ofrece de establecer una herramienta accesible para esta toma de decisiones permite que se incorporen al mercado productos con una consideración medioambiental más sostenible y respetuosa, los que, además, aportan variedad a soluciones habituales para la construcción de muros de cerramiento no portantes. Esta estrategia se podría implementar en el resto de soluciones constructivas y para el resto de productos que se están generando con criterios respetuosos con el medio ambiente, que pueden suponer elementos de desconfianza para los técnicos de la edificación.

De forma particular, de los resultados del EVC en los 28 casos de estudio, se puede extraer que:

- La calidad del BT, según las categorías establecidas para los indicadores, condiciona estrechamente la viabilidad constructiva de una solución arquitectónica. Los resultados demuestran que, cuando el BT no dispone de exigencias de durabilidad certificadas/declaradas (en cuanto a resistencia a ciclos de humectación/ secado, a la erosión, a ciclos de hielo/deshielo - absorción de agua por capilaridad) y está expuesto a condiciones desfavorables, los GI indican que el diseño debe ser revisado para la adecuada disposición constructiva.

- Los valores establecidos para los pesos y combinaciones son válidos para un amplio contexto geográfico, al haber sido diseñados por expertos internacionales, aunque podrían adaptarse para otras situaciones no consideradas.

- Al analizar las tres categorías de indicadores fijados, se destaca que el de requisitos constructivos aporta la mayor proporción de indicadores decisivos para el diseño de la estructura estable del muro. 
- A partir de los indicadores empleados, se verifica que, como en cualquier diseño de fábrica, el arranque en la base o su unión a cimentación, el acabado de la cara exteriory el diseño de huecos son los puntos singulares donde se acumulan ponderaciones más decisivas y, por lo tanto, son aspectos a cuidar para obtener el mejor grado de idoneidad.

En síntesis, se concluye que este EVC puede servir como recurso básico para tomar decisiones en proyectos de obras nuevas o reformas de edificios donde se quiera emplear BT. Para desarrollar un conjunto de criterios de mayor aplicabilidad, se debe considerar indicadores económicos, medioambientales o sociales, que podrían ser incluidos en un procedimiento metodológico complementario al aquí presentado.

\section{AGRADECIMIENTOS}

En primer lugar, se agradece a Daniel Maskell, Guillermo Rolón, Miguel Rocha, Rubén Salvador Roux Gutiérrez y Félix Jové su inestimable interés por aportar su visión crítica y técnica. Asimismo, a todos los técnicos, constructores, empresas y particulares, especialmente al arquitecto Gabriel Barbeta, por facilitar información crucial sobre todos los casos a nivel nacional que han posibilitado el desarrollo de esta herramienta.

\section{REFERENCIAS BIBLIOGRÁFICAS}

AENOR (2009). UNE-ISO/TS 21929-1. Sostenibilidad en construcción de edificios. Indicadores de sostenibilidad. Parte 1: Marco para el desarrollo de indicadores para edificios.

ASTM D559 (1989). Standard Test Methods for Wetting and Drying Compacted Soil-Cement Mixtures. ASTM International.

Canivell, J., Rodríguez-García, R., González-Serrano, A. M. y Romero Girón, A. (2020). Assessment of heritage rammedearth buildings. The Alcázar of King Don Pedro I (Spain). Journal of Architectural Engineering, 6(2). DOI: https://doi. org/10.1061/(ASCE)AE.1943-5568.0000400

Cid-Falceto, J., Mazarrón, F. R. y Cañas, I. (2011). Las normativas de construcción con tierra en el mundo, 63(523), 159-169. Informes de la construcción. DOI: https://doi. org/10.3989/ic.10.011

Deboucha, S. y Hashim, R. (2011). A review on bricks and stabilized compressed earth blocks. Scientific Research and Essays, 6(3), 499-506.

Fernandes, J., Peixoto, M., Mateus, R. y Gervásio, H. (2019). Life cycle analysis of environmental impacts of earthen materials in the Portuguese context: Rammed earth and compressed earth blocks. Journal of Cleaner Production, 241. DOI: https://doi.org/10.1016/j.jclepro.2019.118286
Gandia, R. M., Gomes, F. C., Corrêa, A. A. R., Rodrigues, M. C. y Mendes, R. F. (2019). Physical, mechanical and thermal behavior of adobe stabilized with glass fiber reinforced polymer waste. Construction and Building Materials, 222, 168-182. DOI: https://doi.org/10.1016/j. conbuildmat.2019.06.107

Guettala, A., Abibsi, A. y Houari, H. (2006). Durability study of stabilized earth concrete under both laboratory and climatic conditions exposure. Construction and Building Materials, 20(3), 119-127. DOI: https://doi.org/10.1016/j. conbuildmat.2005.02.001

Jové Sandoval, F., Muñoz de la Calle, D. y Pahíno Rodríguez, L. (2011). Ensayos de erosión hídrica sobre muros de tierra (fábrica de BTC). Método, resultados y discusión. En Construcción con tierra. Tecnología y Arquitectura. Congresos de Arquitectura de Tierra. (pp. 193-204). Valladolid: Cátedra Juan de Villanueva. Universidad de Valladolid.

Lavie Arsène, M.-I., Frédéric, C. y Nathalie, F. (2020). Improvement of lifetime of compressed earth blocks by adding limestone, sandstone and porphyry aggregates. Journal of Building Engineering, 29. DOI: https://doi. org/10.1016/j.jobe.2019.101155

López-Zambrano, M. J., Canivell, J. y Calama-González, C. (2019). Sistema de evaluación de soluciones de rehabilitación energética para edificios bien de interés cultural (SESREBIC). Su aplicación a monasterios BIC. Informes de la Construcción, 71(555), e300. DOI: https://doi.org/10.3989/ic.63532

Mahmood, O. I., Habeeb, A. A. y Al-Jumaili, H. T. (2019). Characteristic properties of CEB made of gypsum soil. International Journal of Advanced Science and Technology, 28(12), 36-45.

Maldonado Ramos, L., Castilla Pascual, F., Vela Cossío, F. y Rivera Gómez, D. (2001). Rendimiento y coste energético en la construcción de cerramientos de fábrica de adobe y bloque de tierra comprimida. Informes de la Construcción, 53(473). DOI: https://doi.org/10.3989/ic.2001.v53.i473.669

Miloudi, Y., Fezzioui, N., Labbaci, B., Benidir, A., Roulet, C. A. y Oumeziane, Y. A. (2019). Hygrothermal characterization of compressed and cement stabilized earth blocks. International Review of Civil Engineering, 10(4), 177-187. DOI: https:// doi.org/10.15866/irece.v10i4.15975

Ministerio de Fomento (1999). Ley de Ordenación de la Edificación (Ley 38/1999). Madrid: Oficial del Estado. España. Recuperado de https://www.boe.es/eli/es/l/1999/11/05/38/ con

Molar-Orozco, M., Velázquez-Lozano, J. y Vázquez-Jimánez, M. (2020). Comportamiento térmico de tres prototipos en Saltillo, Coahuila (bloques de tierra, concreto y tapa de huevo). Hábitat Sustentable, 10(1), 22 - 31. DOI: https://doi. org/10.22320/07190700.2020.10.01.02

Mosquera, P., Canas, I., Cid-Falceto, J. y Marcos, F. (2014). Determination of the thermal conductivity in adobe with several models. Journal of Heat Transfer, 136(3). DOI: https:// doi.org/10.1115/1.4025560 
Soronis, G. (1992). The problem of durability in building design. Construction and Building Materials, 6(4), 205-211. DOI: https://doi.org/10.1016/0950-0618(92)90039-2

UNE 41410 (2008). Bloques de tierra comprimida para muros y tabiques. Definiciones, especificaciones y métodos de ensayo. Madrid: Ministerio de Industria, Turismo y Comercio.

Walker, P. (2001). The Australian Earth building handbook. Sydney, Australia: Standards Australia International.

Wati, E., Bidoung, J. C., Damfeu, J. C. y Meukam, P. (2020). Energy performance of earthen building walls in the equatorial and tropical climates: a case study of Cameroon. Energy Efficiency, 13, 735-750. DOl: https://doi.org/10.1007/s12053020-09856-6 